Pacific Journal of Mathematics

HOLOMORPHIC REPRODUCING KERNELS IN REINHARDT 


\title{
HOLOMORPHIC REPRODUCING KERNELS IN REINHARDT DOMAINS
}

\author{
HAROLD P. BOAS
}

\begin{abstract}
The orthogonal projection $P_{0}: L^{2}(\Omega) \rightarrow L^{2}(\Omega) \cap$ \{holomorphic functions $\}$ (the Bergman projection) is studied, together with its analogue $P_{s}: W^{s}(\Omega) \rightarrow W^{s}(\Omega) \cap\{$ holomorphic functions\}, for smooth bounded pseudoconvex complete Reinhardt domains $\Omega \subset \mathbf{C}^{n}$. It is shown that $P_{s}$ maps the Sobolev space $W^{r}(\Omega)$ boundedly into itself for each $r \geq s$. Explicit formulas are computed for the representing kernel functions for the case of the ball.
\end{abstract}

The Bergman projection $P_{0}$ for a bounded domain in $\mathbf{C}^{n}$, along with the associated Bergman kernel function, has proved fundamental for the theory of boundary behavior of holomorphic mappings: see [3, 6] and their references. In this paper we study the analogous projection $P_{s}$ from a Sobolev space $W^{s}$ onto its holomorphic subspace, and the corresponding reproducing kernel function $K_{s}(w, z)$, in the case of Reinhardt (multi-circular) domains. (See $\$ 1$ for definitions.) In $\S 2$ we compute explicit formulas for this new kernel function in the ball. A notable feature is the appearance of a logarithmic term, even in the simplest case:

TheOrem 2.1. For the unit ball in $\mathbf{C}^{2}$

$$
K_{1}(w, z)=\pi^{-2}\left[(1-\langle w, z\rangle)^{-1}+\langle w, z\rangle^{-1} \log (1-\langle w, z\rangle)^{-1}\right]
$$

where $\langle w, z\rangle=\sum w_{j} \bar{z}_{j}$ is the usual hermitian scalar product. For comparison we recall that the usual Bergman kernel for the unit ball in $\mathbf{C}^{n}$ is

$$
K_{0}(w, z)=n ! \pi^{-n}(1-\langle w, z\rangle)^{-n-1} .
$$

The "main term" of the kernel $K_{s}(w, z)$ turns out to be

$$
(n-2 s) ! \pi^{-n}(1-\langle w, z\rangle)^{-n-1+2 s} \text { if } 2 s \leq n ;
$$

when $2 s \geq n+1$ the leading term involves $\log (1-\langle w, z\rangle)$. Theorem 2.3 contains the details.

S. R. Bell and the author showed [5] that the Bergman projection $P_{0}$ for a smooth bounded complete Reinhardt domain $\Omega \subset \mathbf{C}^{n}$ is bounded on $C^{\infty}(\bar{\Omega})$; in other words for each $t \geq 0$ there is $N=N(t) \geq 0$ such that $P_{0}$ 
is bounded from $W^{t+N}$ into $W^{t}$ (see also [2]). Using a different method we now show that $N$ may be taken equal to 0 if $\Omega$ is additionally a domain of holomorphy; moreover the projection $P_{s}$ admits the same property.

THEOREM 3.1. Let $\Omega \subset \mathbf{C}^{n}$ be a smooth bounded pseudoconvex complete Reinhardt domain. Then (a) the Bergman projection maps $W^{t}(\Omega)$ boundedly into itself for every $t \geq 0$, and, more generally, (b) for each integer $s \geq 0$ the orthogonal projection $P_{s}$ from the Sobolev space $W^{s}(\Omega)$ onto its holomorphic subspace maps $W^{t}(\Omega)$ boundedly into itself for every $t \geq s$.

We give two proofs of this theorem. In $\S 3$ we obtain the result as a corollary of J. J. Kohn's theory of the $\bar{\partial}$-Neumann problem with weights. The second proof, presented in $\S 4$, is elementary in spirit (but valid only for the convex case). In $\$ 5$ we discuss a key ingredient of the second method, an integral inequality for convex domains pointing in the opposite direction to the Cauchy-Schwarz inequality; it has some intrinsic interest.

A second lemma of independent interest, which holds for any smooth bounded domain, says roughly that we can integrate by parts with no boundary term if the integrand has a holomorphic factor.

LEMMA 6.1. (Holomorphic integration by parts.) Let $\Omega \subset \mathbf{C}^{n}$ be a smooth bounded domain. For each $j, 1 \leq j \leq n$, there is a linear differential operator

$$
L_{j}=a_{0}+\sum_{k=1}^{n}\left(a_{j k} \frac{\partial}{\partial z_{k}}+b_{\jmath k} \frac{\partial}{\partial \bar{z}_{k}}\right),
$$

the a's and b's being functions in $C^{\infty}(\bar{\Omega})$, such that for all sufficiently regular functions $f$ and $h$, with h holomorphic,

$$
\int_{\Omega} f \frac{\overline{\partial h}}{\partial z}=\int_{\Omega}\left(L_{J} f\right) h .
$$

(It suffices if $f, h \in W^{1}(\Omega)$.)

Section 6 contains the proof, based on a remark of J. J. Kohn.

I thank my thesis advisor Norberto Kerzman, who suggested study of the $K_{s}$ kernel. The computations of $\S 2$ for the ball and the holomorphic integration by parts lemma are based on a part of my thesis, as is the discussion of interpolation spaces; the remainder of $\S 3$ and $\S \S 4$ and 5 are new. 
1. Preliminaries. Let $\Omega \subset \mathbf{C}^{n}$ be a bounded domain. The space of complex-valued functions on $\Omega$ with square-integrable (distribution) derivatives through order $s$ is denoted $W^{s}(\Omega)$ or simply $W^{s}$. Provided with the scalar product

$$
\langle f, g\rangle_{s}=\sum_{|\alpha|+|\beta| \leq s} \int_{\Omega} \frac{\partial^{\beta}}{\partial \bar{z}^{\beta}} \frac{\partial^{\alpha} f}{\partial z^{\alpha}} \frac{\partial^{\beta}}{\partial z^{\beta}} \frac{\partial^{\alpha} \bar{g}}{\partial \bar{z}^{\alpha}} d m
$$

and the norm $\|f\|_{s}^{2}=\langle f, f\rangle_{s}, W^{s}$ is a Hilbert space. Here $d m$ is Lebesgue measure and $\alpha$ and $\beta$ are multi-indices. When $s=0$ we regain the usual $L^{2}$ scalar product - indeed, $W^{0}=L^{2}$. The subspace of those functions in $W^{s}$ which are holomorphic is denoted $H^{s}(\Omega)$.

Warning. Often $H^{s}$ is used in the literature for the Sobolev space we call $W^{s}$. We always reserve the letter $H$ for spaces of holomorphic functions.

By the mean-value property

$$
|f(z)| \leq\|f\|_{0} \gamma_{n}^{-1 / 2} d(z)^{-n} \quad \text { for } z \in \Omega, f \in H^{s},
$$

where $\gamma_{n}$ is the volume of the unit ball and $d(z)$ is the Euclidean distance from $z$ to the boundary of $\Omega$. It follows first that $H^{s}$ is a closed subspace of $W^{s}$ and second that the point evaluation functional $f \rightarrow f(z)$ is bounded in $H^{0}$ and $a$ fortiori in $H^{s}$.

By the general theory of reproducing kernels $[1,8]$ it follows that there is a kernel function $K_{s}(w, z)$ with the following properties:

(1) for $z$ fixed, $K_{s}(\cdot, z) \in H^{s}$;

(2) $K_{s}(w, z)=\overline{K_{s}(z, w)}$;

(3) $P_{s} f(w)=\left\langle f(\cdot), K_{s}(\cdot, w)\right\rangle_{s}, f \in W^{s}$, where $P_{s}: W^{s} \rightarrow H^{s}$ is the orthogonal projection;

(4) $K_{s}(w, z)=\Sigma \varphi_{j}(w) \bar{\varphi}_{j}(z) /\left\|\varphi_{j}\right\|_{s}^{2}$ where $\left\{\varphi_{J}\right\}$ is a complete orthogonal system for $H^{s}$;

(5) the norm of the point evaluation functional at $z$ is

$$
\left(\sum\left|\varphi_{j}(z)\right|^{2} /\left\|\varphi_{J}\right\|_{s}^{2}\right)^{1 / 2} \text {. }
$$

Hence the sum in (4) converges absolutely and uniformly on compact subsets of $\Omega \times \Omega$.

The kernel $K_{s}$ has also been mentioned by S. R. Bell [4].

A complete Reinhardt (or multi-circular) domain is one which, whenever it contains a point $\left(z_{1}, \ldots, z_{n}\right)$, also contains all points $\left(\lambda_{1} z_{1}, \ldots, \lambda_{n} z_{n}\right)$ with $\left|\lambda_{j}\right| \leq 1, j=1, \ldots, n$. In such a domain the monomials $z^{\alpha}$ form an orthogonal system in both $H^{0}$ and $H^{s}$, as follows by $n$-fold integration in polar coordinates; they also form a complete system since 
every holomorphic function in a complete Reinhardt domain has a power series expansion valid throughout the domain. By property (4) the $H^{s}$ reproducing kernel takes the form

$$
K_{s}(w, z)=\sum_{\alpha} \frac{w^{\alpha} \bar{z}^{\alpha}}{\left\|z^{\alpha}\right\|_{s}^{2}} .
$$

A bounded domain $\Omega \subset \mathbf{C}^{n}$ is called smooth if there is a $C^{\infty}$ defining function $\rho: \mathbf{C}^{n} \rightarrow \mathbf{R}$ such that $\Omega=\{z: \rho(z)<0\}$, the boundary $b \Omega=\{z$ : $\rho(z)=0\}$, and the gradient of $\rho$ does not vanish on $b \Omega$.

2. The $H^{s}$ reproducing kernel for the ball. In this section we compute $K_{s}$ by formula (1.1) for the special case of $\Omega=B_{n}$, the unit ball in $\mathbf{C}^{n}$. It is easy to see that any unitary transformation $U$ preserves the $H^{s}\left(B_{n}\right)$ scalar product. Hence $K_{s}$ has the invariance property $K_{s}(U w, U z)$ $=K_{s}(w, z)$. If for a fixed $w \in B_{n}$ we choose a unitary $U$ such that $U w$ has only its first coordinate non-zero, we obtain

$$
K_{s}(w, z)=\sum_{k=0}^{\infty} \frac{(\langle w, z\rangle)^{k}}{\left\|z_{1}^{k}\right\|_{s}^{2}} .
$$

It is a straightforward induction, using the one-dimensional formula

$$
\int_{0}^{1} x^{a}(1-x)^{b} d x=\Gamma(a+1) \Gamma(b+1) / \Gamma(a+b+2),
$$

to show that

(see e.g. [15, page 49]).

$$
\left\|z_{1}^{k}\right\|_{w^{0}\left(B_{n}\right)}^{2}=\pi^{n} \frac{k !}{(k+n) !}
$$

Since the $H^{s}$ norm is the sum of the $W^{0}$ norms of the derivatives, it follows that, with $m=\min (k, s)$,

$$
\begin{aligned}
\left\|z_{1}^{k}\right\|_{s}^{2}=\left\|z_{1}^{k}\right\|_{0}^{2}+\sum_{j=1}^{m} k^{2}(k-1)^{2} \cdots(k-j+1)^{2}\left\|z_{1}^{k-\jmath}\right\|_{0}^{2} \\
=\pi^{n}\left[\frac{k !}{(n+k) !}+\sum_{j=1}^{m} k^{2}(k-1)^{2}\right. \\
\left.\cdots(k-j+1)^{2} \frac{(k-j) !}{(n+k-j) !}\right] \\
=\pi^{n} \frac{k !}{(n+k) !}\left[1+\sum_{j=1}^{s} k(n+k)(k-1)(n+k-1)\right. \\
\cdots(k-j+1)(n+k-j+1)] .
\end{aligned}
$$


It is legitimate to extend the final summation all the way from 1 to $s$ because the supplementary terms (if any) are all zero. Thus

$$
\left\|z_{1}^{k}\right\|_{s}^{2}=\pi^{n} \frac{k !}{(n+k) !} p_{s}(k)
$$

where $p_{s}$ is a monic polynomial of degree $2 s$.

The series (2.1) now takes the form

$$
K_{s}(w, z)=F_{s}(\langle w, z\rangle)
$$

where

$$
F_{s}(x)=\pi^{-n} \sum_{k=0}^{\infty} \frac{(n+k) !}{k !} \frac{x^{k}}{p_{s}(k)}, \quad|x|<1 .
$$

THEOREM 2.1. The $H^{1}$ reproducing kernel for the unit ball in $\mathbf{C}^{2}$ is

$$
K_{1}(w, z)=\pi^{-2}\left[\frac{1}{1-\langle w, z\rangle}+\frac{1}{\langle w, z\rangle} \log \frac{1}{1-\langle w, z\rangle}\right]
$$

where we take the principal branch of the natural logarithm and define $x^{-1} \log (1-x)^{-1}$ at $x=0$ to be equal to its limit 1 .

For the proof we sum the series $(2.3)$ with $p_{1}(k)=1+k(2+k)=$ $(k+1)^{2}$ :

$$
\begin{aligned}
\pi^{2} F_{1}(x) & =\sum \frac{(2+k) !}{k !} \frac{x^{k}}{(k+1)^{2}}=\sum \frac{2+k}{1+k} x^{k} \\
& =\sum x^{k}+\sum \frac{x^{k}}{1+k}=\frac{1}{1-x}+\frac{1}{x} \log \frac{1}{1-x},
\end{aligned}
$$

which is the desired result.

For $n>2$ the series has the following integral representation.

THEOREM 2.2. The $H^{1}$ reproducing kernel for the unit ball in $\mathbf{C}^{n}, n>2$, is

$$
K_{1}(w, z)=\frac{n !}{\pi^{n}} \frac{1}{\sqrt{\left(n^{2}-4\right) / 4}} \int_{0}^{\infty} \frac{e^{-n t / 2} \sinh \sqrt{\left(n^{2}-4\right) / 4} t}{\left(1-e^{-t}\langle w, z\rangle\right)^{n+1}} d t
$$

Proof. In this case the series (2.3) becomes

$$
\pi^{n} F_{1}(x)=\sum \frac{(n+k) !}{k !} \frac{x^{k}}{k^{2}+n k+1} .
$$


The rational function $\left(k^{2}+n k+1\right)^{-1}$ is the Laplace transform of

$$
\left[\sqrt{\left(n^{2}-4\right) / 4}\right]^{-1} e^{-n t / 2} \sinh \sqrt{\left(n^{2}-4\right) / 4} t,
$$

so

$$
\begin{aligned}
\pi \sqrt[n]{\left(n^{2}-4\right) / 4} F_{1}(x) \\
\quad=\sum_{k=0}^{\infty} \frac{(n+k) !}{k !} \int_{0}^{\infty} x^{k} e^{-k t} e^{-n t / 2} \sinh \sqrt{\left(n^{2}-4\right) / 4} t d t \\
\quad=\int_{0}^{\infty} \sum_{0}^{\infty} \frac{(n+k) !}{k !}\left(x e^{-t}\right)^{k} e^{-n t / 2} \sinh \sqrt{\left(n^{2}-4\right) / 4} t d t \\
=n ! \int_{0}^{\infty} \frac{e^{-n t / 2} \sinh \sqrt{\left(n^{2}-4\right) / 4} t}{\left(1-x e^{-t}\right)^{n+1}} d t .
\end{aligned}
$$

The exchange of summation and integration is justified because

$$
\begin{gathered}
\int_{0}^{\infty} e^{-k t} e^{-n t / 2} \sinh \sqrt{\left(n^{2}-4\right) / 4} t d t \\
\quad \leq \int_{0}^{\infty} e^{-n t / 2} \sinh \sqrt{\left(n^{2}-4\right) / 4} t d t \\
\quad \leq \int_{0}^{\infty} e^{-a t} d t<\infty
\end{gathered}
$$

where $a=n / 2-\sqrt{\left(n^{2}-4\right) / 4}>0$.

In general the series (2.3) does not admit a simple closed form representation, but the main term is not hard to determine.

THEOREM 2.3. The reproducing kernel $K_{s}(w, z)$ for the unit ball $B_{n}$ is represented by the following asymptotic series:

$$
\begin{gathered}
K_{s}(w, z)-\frac{(n-2 s) !}{\pi^{n}} \frac{1}{(1-\langle w, z\rangle)^{n+1-2 s}} \\
\quad \times[1+(1-\langle w, z\rangle) \times \text { polynomial in }\langle w, z\rangle] \\
\quad \sum_{J=0}^{\infty} c_{J}(s)(1-\langle w, z\rangle)^{\prime} \log (1-\langle w, z\rangle) \quad \text { if } 2 s \leq n ; \\
K_{s}(w, z)-\frac{(-1)^{n}}{\pi^{n}(2 s-n-1) !}(1-\langle w, z\rangle)^{2 s-n-1} \log (1-\langle w, z\rangle) \\
\sim \sum_{J=2 s-n}^{\infty} c_{J}^{\prime}(s)(1-\langle w, z\rangle)^{j} \log (1-\langle w, z\rangle) \quad \text { if } 2 s \geq n+1 .
\end{gathered}
$$


The symbol $\sim$ means that if the series is terminated with the $m$ th power then the difference between the right-hand and left-hand sides is an analytic function of the single variable $\langle w, z\rangle$ which is bounded in $\{|\langle w, z\rangle|<1\}$ together with all derivatives through order $m$.

REMARK. The asymptotic expansion of C. Fefferman [12] for the usual Bergman kernel on a strongly pseudoconvex domain contains a logarithmic term in general, but this term vanishes for the ball. Theorem 2.3 shows, in particular, that for large enough $s$ the $K_{s}$ kernel inevitably contains a logarithmic term.

To prove the theorem we have to determine the behavior of (2.3). The result follows immediately from an elementary

LEMMA. Let $S(x)=\sum_{k=i}^{\infty} x^{k} q(k) / r(k)$ where $q$ and $r$ are monic polynomials of degrees $a$ and $b$, respectively, with $r(k) \neq 0$ for $k \geq i$. Then $S(x)$ represents an analytic function in the unit disk with the following property:

$$
\begin{gathered}
S(x)-\frac{(a-b) !}{(1-x)^{a-b+1}}\left[1+\alpha_{1}(1-x)+\cdots+\alpha_{a-b}(1-x)^{a-b}\right] \\
\sim \sum_{j=0}^{\infty} c_{j}(1-x)^{j} \log (1-x) \quad \text { if } b \leq a \\
S(x)-\frac{(-1)^{b-a}}{(b-a-1) !}(1-x)^{b-a-1} \log (1-x) \\
\sim \sum_{j=b-a}^{\infty} c_{j}^{\prime}(1-x)^{j} \log (1-x) \quad \text { if } b \geq a+1 .
\end{gathered}
$$

Once again $\sim$ means that if the series is stopped with the $m$ th power then the remainder represents an analytic function on $\{|x|<1\}$ bounded together with all derivatives through order $m$.

Proof. If $b \geq a+2$ then the series for $S(x)$ is uniformly majorized by the convergent series $\Sigma 1 / k^{2}$. Hence all derivatives of $S$ of order $b-a-2$ or less are bounded analytic functions in the unit disk.

For $b \geq a+1$ set

$$
\frac{q(k)}{r(k)}=\frac{q_{1}(k)}{r_{1}(k)}+\prod_{j=0}^{b-a-1}(k-j)^{-1}
$$


with $\operatorname{deg} r_{1}-\operatorname{deg} q_{1}>\operatorname{deg} r-\operatorname{deg} q$. Since

$$
\begin{aligned}
(b- & a-1) ! \sum_{b-a}^{\infty} \frac{x^{k}(k-b+a) !}{k !} \\
& =(-1)^{b-a}(1-x)^{b-a-1} \log (1-x)+(\text { polynomial in } x)
\end{aligned}
$$

the second part follows by induction.

For $b \leq a$ set

$$
\frac{q(k)}{r(k)}=\frac{q_{2}(k)}{r(k)}+\prod_{j=1}^{a-b}(k+j)
$$

with $\operatorname{deg} q_{2}<\operatorname{deg} q$. Since

$$
\sum_{0}^{\infty} \frac{x^{k}(k+a-b) !}{k !}=\frac{(a-b) !}{(1-x)^{a-b+1}},
$$

the first part follows by induction and by the second part.

3. The projection $P_{s}$ in Reinhardt domains. It is well known that on smooth bounded strongly pseudoconvex domains the Bergman projection preserves each Sobolev space $W^{r}, r \geq 0$. The same is true more generally for those smooth bounded weakly pseudoconvex domains which admit sub-elliptic estimates for the $\bar{\partial}$-Neumann problem. Reinhardt domains, on the other hand, can have complex varieties in the boundary, which means that sub-elliptic estimates cannot hold in general. Nonetheless we get optimal regularity estimates for $P_{0}$ and also for $P_{s}$.

THEOREM 3.1. Let $\Omega \subset \mathbf{C}^{n}$ be a smooth bounded (weakly) pseudoconvex complete Reinhardt domain. Then (a) the Bergman projection $P_{0}: W^{0}(\Omega) \rightarrow$ $H^{0}(\Omega)$ is bounded from $W^{r}(\Omega)$ to $H^{r}(\Omega)$ for every real number $r \geq 0$; and more generally (b) for each integer $s \geq 0$ the orthogonal projection $P_{s}$ : $W^{s}(\Omega) \rightarrow H^{s}(\Omega)$ is bounded from $W^{r}(\Omega)$ to $H^{r}(\Omega)$ for every real number $r \geq s$.

Proof. The proof is based on the regularity estimates of J. J. Kohn [14] for the $\bar{\partial}$-Neumann problem with weights on a smooth bounded (weakly) pseudoconvex domain. (However, we give another proof in the next section.) It is a consequence of Kohn's work that given an integer $r \geq 0$ there exists $T \geq 0$ such that for all $t>T$ the orthogonal projection $P_{0}^{(t)}$ from the weighted space $W^{0}\left(\Omega, \exp \left(-t|z|^{2}\right) d m(z)\right)=W_{(t)}^{0}$ onto the subspace of holomorphic functions is bounded from $W^{r}(\Omega, d m)$ to $H^{r}(\Omega, d m)$. Here $d m$ is Lebesgue measure and an index in parentheses refers to an object relative to the weighted norm. 
When $\Omega$ is a complete Reinhardt domain the usual Bergman projection $P_{0}$ and the weighted projection $P_{0}^{(t)}$ are related in a particularly simple way. The weight function $\exp \left(-t|z|^{2}\right)$ has multi-circular symmetry, so the monomials $z^{\alpha}$ are orthogonal with respect to the weight as well as in the usual norms. Accordingly

$$
P_{0}^{(t)} f(w)=\int_{\Omega} K_{0}^{(t)}(w, z) f(z) \exp \left(-t|z|^{2}\right) d m
$$

where

$$
K_{0}^{(t)}(w, z)=\sum_{\alpha} \frac{w^{\alpha} \bar{z}^{\alpha}}{\left\|z^{\alpha}\right\|_{(t)}^{2}} .
$$

Let $\mathfrak{R}$ denote the multiplication operator

$$
\Re \sum_{\alpha} C_{\alpha} z^{\alpha}=\sum_{\alpha} \frac{C_{\alpha} z^{\alpha}\left\|z^{\alpha}\right\|_{(t)}^{2}}{\left\|z^{\alpha}\right\|^{2}} .
$$

Since $\exp \left(-t|z|^{2}\right) \leq 1$, we have $\left\|z^{\alpha}\right\|_{(t)}^{2} \leq\left\|z^{\alpha}\right\|^{2}$, so $\Re$ is bounded (with norm $\leq 1$ ) from $H^{r}$ to $H^{r}$ for every integer $r \geq 0$. Now

$$
P_{0} f(w)=\operatorname{N} P_{0}^{(t)}\left(f(\cdot) \exp t|\cdot|^{2}\right)(w),
$$

so by the cited result of Kohn,

$$
\left\|P_{0} f\right\|_{r} \leq\left\|P_{0}^{(t)}\left(f(\cdot) \exp t|\cdot|^{2}\right)\right\|_{r} \leq C\left\|f(\cdot) \exp t|\cdot|^{2}\right\|_{r} \leq C^{\prime}\|f\|_{r}
$$

where the constants are independent of $f$. This proves that the Bergman projection $P_{0}$ is bounded from $W^{r}$ to $H^{r}$ for each integer $r \geq 0$.

To extend this result to non-integral $r$ we have first of all to define Sobolev spaces of non-integral order. This is done by interpolation. We digress briefly to remind the reader of a simple method [16] for defining intermediate Hilbert spaces. We need only a special case of an elaborate theory; for a discussion of the general case see e.g. [7].

Given two separable Hilbert spaces $X$ and $Y$ and a continuous inclusion $i: X \rightarrow Y$ with dense image there is a positive (unbounded) self-adjoint operator $\Lambda$ on $Y$ such that $X$ is the domain of $\Lambda$ and $\|x\|_{X}=\|\Lambda x\|_{Y}$. Define the intermediate space $[X, Y]_{\theta}$ for $0 \leq \theta \leq 1$ as the domain of $\Lambda^{1-\theta}$ with norm equivalent to $\left\|\Lambda^{1-\theta} x\right\|_{Y}$.

In the case at hand $X$ and $Y$ are Sobolev spaces of different orders, so the inclusion $i$ is compact (by the Rellich lemma). Then $i i^{*}: Y \rightarrow Y$ is a positive compact self-adjoint operator and we may take $\Lambda=\left(i i^{*}\right)^{-1 / 2}$. In 
particular if $\left\{\varphi_{j}\right\}$ is an orthonormal basis for $Y$ of eigenvectors of $i i^{*}$ we have the representations

$$
Y=\left\{\sum a_{j} \varphi_{j}: \sum\left|a_{j}\right|^{2}<\infty\right\}, \quad X=\left\{\sum a_{j} \varphi_{j}: \sum\left|a_{j}\right|^{2} \lambda_{j}^{2}<\infty\right\},
$$

and

$$
[X, Y]_{\theta}=\left\{\sum a_{j} \varphi_{j}: \sum\left|a_{j}\right|^{2} \lambda_{J}^{2(1-\theta)}<\infty\right\},
$$

where $\lambda_{J}=\left\|\varphi_{j}\right\|_{X}$. When $X$ and $Y$ are Sobolev spaces of holomorphic functions in a complete Reinhardt domain, we may take for the system $\left\{\varphi_{j}\right\}$ the monomials $z^{\alpha}$ suitably normalized.

One of the fundamental principles of interpolation theory is that if $X_{1}, Y_{1}$ and $X_{2}, Y_{2}$ are two pairs of Hilbert spaces as described and if $L$ is a linear operator which is bounded from $Y_{1}$ to $Y_{2}$ and bounded from $X_{1}$ to $X_{2}$ then $L$ is bounded from $\left[X_{1}, Y_{1}\right]_{\theta}$ to $\left[X_{2}, Y_{2}\right]_{\theta}, 0 \leq \theta \leq 1$. It is well-known for the usual Sobolev spaces $W^{s}$ on a smooth bounded domain that $\left[W^{s}, W^{r}\right]_{\theta}=W^{\theta r+(1-\theta) s}$ when $s, r$, and $\theta r+(1-\theta) s$ are integers, $0 \leq r \leq s, 0 \leq \theta \leq 1$, in the sense that the two spaces have the same elements and equivalent norms. When $\theta r+(1-\theta) s$ is not an integer the preceding equation defines the space on the right-hand side. The following natural observation does not seem to have been made before.

THEOREM 3.2. Let $\Omega \subset \mathbf{C}^{n}$ be a smooth bounded domain of holomorphy (not necessarily Reinhardt). Let $H^{s}$ be the subspace of holomorphic functions contained in the Sobolev space $W^{s}$. Then

$$
\left[H^{s}, H^{r}\right]_{\theta}=H^{\theta r+(1-\theta) s}
$$

with equivalent norms, where $0 \leq r \leq s, 0 \leq \theta \leq 1$.

Proof. It suffices to consider the case of $r$ and $s$ integral, the general case following by the reiteration theorem. Considering our definition of intermediate spaces we should note that $H^{s}$ is dense in $H^{r}$; in fact, David Catlin has shown [9] the stronger statement that $C^{\infty}(\bar{\Omega}) \cap$ (holomorphic functions) is dense in $H^{r}$ for every integer $r \geq 0$.

Fixing $r$ and $s$ we know from Kohn that for sufficiently large $t$ the weighted projection $P_{0}^{(t)}$ is bounded from $W^{r}$ to $H^{r}$ and from $W^{s}$ to $H^{s}$, and therefore by interpolation it is bounded from $\left[W^{s}, W^{r}\right]_{\theta}=W^{\theta r+(1-\theta) s}$ to $\left[H^{s}, H^{r}\right]_{\theta}$. But $P_{0}^{(t)}$ is the identity on holomorphic functions, so the identity maps $H^{\theta r+(1-\theta) s}$ continuously into $\left[H^{s}, H^{r}\right]_{\theta}$. Conversely, the inclusion is bounded from $H^{r}$ into $W^{r}$ and from $H^{s}$ into $W^{s}$, hence from 
$\left[H^{s}, H^{r}\right]_{\theta}$ into $W^{\theta r+(1-\theta) s}$. Thus the identity also maps $\left[H^{s}, H^{r}\right]_{\theta}$ continuously into $H^{\theta r+(1-\theta) s}$. Therefore the two spaces coincide and have equivalent norms.

The author does not know the answer to the next question.

Question 3.3. Does the preceding theorem hold if $\Omega$ is not a domain of holomorphy?

What we needed in the proof of Theorem 3.2 was the boundedness from $W^{r}$ to $H^{r}$ for all $r$ of some projection - it could be the Bergman projection, a weighted projection, or even one of the Sobolev space projections.

Proof of Theorem 3.1 concluded. We showed statement (a) for integral $r$; the case of real $r$ follows by interpolation. It remains to prove statement (b) for $s \geq 1$.

Since the monomials $z^{\alpha}$ are a complete orthogonal set in $H^{r}$ for every $r \geq 0$ equation (3.1) implies the estimate

$$
\left\|z^{\alpha}\right\|_{r}^{\theta}\left\|z^{\alpha}\right\|_{t}^{1-\theta} \leq C(r, t, \theta)\left\|z^{\alpha}\right\|_{\theta r+(1-\theta) t}
$$

for $0 \leq \theta \leq 1$, where the constant $C(r, t, \theta)$ is independent of $\alpha$. (An inequality in the other direction holds as well, but we do not need it.)

Consider the operator $U_{s}$ given by

$$
U_{s} h(w)=\int_{\Omega} K_{s}(w, z) h(z) d m
$$

where $h$ is a holomorphic function. (This operator has also been considered by Steve Bell [4] in the setting of smooth bounded strongly pseudoconvex domains.) Supposing, to ensure convergence, that $h(z)=\sum C_{\alpha} z^{\alpha}$ is a holomorphic polynomial, we compute for any $t \geq 0$

$$
\begin{aligned}
\left\|U_{s} h\right\|_{t+2 s}^{2} & =\sum_{\alpha} \frac{\left|C_{\alpha}\right|^{2}\left\|z^{\alpha}\right\|_{0}^{4}\left\|z^{\alpha}\right\|_{t+2 s}^{2}}{\left\|z^{\alpha}\right\|_{s}^{4}} \\
& =\sum_{\alpha}\left|C_{\alpha}\right|^{2}\left\|z^{\alpha}\right\|_{t}^{2}\left(\frac{\left\|z^{\alpha}\right\|_{0}^{4}\left\|z^{\alpha}\right\|_{t+2 s}^{2}}{\left\|z^{\alpha}\right\|_{s}^{4}\left\|z^{\alpha}\right\|_{t}^{2}}\right) \\
& \leq\|h\|_{t}^{2} C\left(0, t+2 s, \frac{t+s}{t+2 s}\right)^{4} C\left(0, t+2 s, \frac{2 s}{t+2 s}\right)^{2}
\end{aligned}
$$

by the interpolation estimate (3.2). Therefore $U_{s}$ is bounded from $H^{t}$ to $H^{t+2 s}$ for every $t \geq 0$. 
To prove (b) we apply the holomorphic integration by parts lemma to write for any $f \in C^{\infty}(\bar{\Omega})$

$$
\begin{aligned}
P_{s} f(w) & =\left\langle f(z), K_{s}(z, w)\right\rangle_{s} \\
& =\sum_{|\alpha| \leq s}\left\langle L^{\alpha} \frac{\partial^{|\alpha|}}{\partial z^{\alpha}} f(z), K_{s}(z, w)\right\rangle_{0} \\
& =\sum_{|\alpha| \leq s} U_{s} P_{0} L^{\alpha} \frac{\partial^{|\alpha|}}{\partial w^{\alpha}} f(w) .
\end{aligned}
$$

Since $\partial^{|\alpha|} / \partial w^{\alpha}$ loses at most $s$ derivatives, $L^{\alpha}$ also loses at most $s$ derivatives, $P_{0}$ breaks even, and $U_{s}$ gains $2 s$ derivatives, we conclude that $P_{s}$ is bounded from $W^{t}$ to $H^{t}$ for every $t \geq 2 s$. By definition $P_{s}$ is bounded from $W^{s}$ to $H^{s}$, so by interpolation we obtain the result for all $t \geq s$. This completes the proof of Theorem 3.1.

4. Regularity of the projection: a second proof. In this section we give another proof of Theorem 3.1, in the special case of a convex Reinhardt domain, which avoids the theory of the $\bar{\partial}$-equation. The tools we use are comparatively unsophisticated, consisting primarily in some inequalities for integrals which ought to be better known.

There are several reasons for presenting this alternative proof. First, it is more aesthetically satisfying to extract a kernel with a nut-cracker than with a sledgehammer. Second, the techniques have independent interest. Third, David Catlin has shown [10], at least for smooth bounded pseudoconvex domains in $\mathbf{C}^{2}$, that regularity estimates in Sobolev norms for the Bergman projection are completely equivalent to such estimates for the Neumann operator; therefore we have an elementary proof of regularity of the $\bar{\partial}$-Neumann problem in the very special case of smooth bounded convex Reinhardt domains in $\mathbf{C}^{2}$.

The precise statement we are going to prove, which is weaker than Theorem 3.1, is the following.

THEOREM 4.1. Let $\Omega \subset \mathbf{C}^{n}$ be a smooth bounded convex Reinhardt domain. Then

(a) the Bergman projection $P_{0}$ is bounded from $W^{r}$ to $H^{r}$ for every real number $r \geq 0$; and more generally

(b) for each integer $s \geq 0$ the projection $P_{s}: W^{s} \rightarrow H^{s}$ is bounded from $W^{r}$ to $H^{r}$ for every real number $r \geq s$.

The key to the proof is the following lemma, which does not involve the smoothness hypothesis. 
LEMMA 4.2. Let $\Omega \subset \mathbf{C}^{n}$ be a bounded convex Reinhardt domain. For each multi-index $\beta$ there is a bounded linear operator $M_{\beta}: H^{0} \rightarrow H^{0}$ such that

$$
\left\langle u, \frac{\partial^{|\beta|}}{\partial z^{\beta}} v\right\rangle_{0}=\left\langle\frac{\partial^{|\beta|}}{\partial z^{\beta}} M_{\beta} u, v\right\rangle_{0}
$$

for all holomorphic polynomials $u$ and $v$.

It is essential not only that $M_{\beta}$ be bounded, but also that it preserve holomorphic functions.

Proof. The operator $M_{\beta}$ is determined by its action on monomials. Setting $u=z^{\alpha}$ and $v=z^{\alpha+\beta}$ in (4.1) we find

$$
M_{\beta} z^{\alpha}=z^{\alpha+2 \beta} \frac{\left\|z^{\alpha}\right\|_{0}^{2}}{\left\|z^{\alpha+\beta}\right\|_{0}^{2}} \frac{(\alpha+\beta) !(\alpha+\beta) !}{\alpha !(\alpha+2 \beta) !}
$$

where $(\alpha+\beta)$ ! means $\left(\alpha_{1}+\beta_{1}\right) !\left(\alpha_{2}+\beta_{2}\right) ! \cdots\left(\alpha_{n}+\beta_{n}\right)$ !, etc. The real content of the lemma is that $M_{\beta}$ is bounded.

If we view $H^{0}$ as a sequence space $l^{2}$ then $M_{\beta}$ acts essentially as a shift operator followed by a multiplication operator. It is enough therefore to show that $M_{\beta}$ is bounded on monomials, i.e. that

$$
\frac{\left\|z^{\alpha}\right\|_{0}\left\|z^{\alpha+2 \beta}\right\|_{0}}{\left\|z^{\alpha+\beta}\right\|_{0}^{2}} \frac{(\alpha+\beta) !(\alpha+\beta) !}{\alpha !(\alpha+2 \beta) !} \leq C_{\beta}
$$

with the bound $C_{\beta}$ independent of $\alpha$. Observing that

$$
\frac{(\alpha+\beta) !(\alpha+\beta) !}{\alpha !(\alpha+2 \beta) !}=\left(\begin{array}{c}
\alpha+\beta \\
\beta
\end{array}\right) /\left(\begin{array}{c}
\alpha+2 \beta \\
\beta
\end{array}\right) \leq 1
$$

we reduce to proving the estimate

$$
\left\|z^{\alpha}\right\|_{0}\left\|z^{\alpha+2 \beta}\right\|_{0} \leq C_{\beta}\left\|z^{\alpha+\beta}\right\|_{0}^{2}
$$

By setting $\gamma=\alpha, \delta=\alpha+2 \beta, \varepsilon=\zeta=\alpha+\beta$ in the following assertion we get (4.2) with $C_{\beta}=16^{n|\beta|}$.

TheOREM 4.3. Let $\Omega \subset \mathbf{C}^{n}$ be a bounded convex Reinhardt domain. For all multi-indices $\gamma, \delta, \varepsilon, \zeta$ such that $\gamma+\delta=\varepsilon+\zeta$ we have

$$
\left\|z^{\gamma}\right\|_{0}\left\|z^{\delta}\right\|_{0} \leq\left\|z^{\varepsilon}\right\|_{0}\left\|z^{\zeta}\right\|_{0} 4^{n|\gamma-\delta|} \text {. }
$$

We defer the proof of Theorem 4.3 to the end of the next section. 
Proof of Theorem 4.1. (a) First suppose $r \geq 0$ is an integer. We have to show that if $f \in W^{r}$ then $P_{0} f \in H^{r}$ and $\left\|P_{0} f\right\|_{r} \leq C\|f\|_{r}$, with the constant $C$ independent of $f$. It is the same to show for an arbitrary multi-index $\beta,|\beta| \leq r$, that

$$
\text { (i) } \frac{\partial^{|\beta|}}{\partial z^{\beta}} P_{0} f \in H^{0} \text { and (ii) }\left\|\frac{\partial^{|\beta|}}{\partial z^{\beta}} P_{0} f\right\|_{0} \leq C\|f\|_{r} \text {. }
$$

Given a holomorphic function $g(z)=\Sigma_{\alpha} c_{\alpha} z^{\alpha}$ we denote the partial sum $\Sigma_{\gamma-\alpha \geq 0} c_{\alpha} z^{\alpha}$ by $S_{\gamma}(g)$, where $\gamma-\alpha \geq 0$ means $\gamma_{J}-\alpha, \alpha_{j} \geq$ for all $j$. Then (i) and (ii) will both follow from the estimate

$$
\left|\left\langle h, \frac{\partial^{|\beta|}}{\partial z^{\beta}} S_{\gamma}\left(P_{0} f\right)\right\rangle_{0}\right| \leq C\|h\|_{0}\|f\|_{r}
$$

for all $\gamma$ and all holomorphic polynomials $h$, where $C$ is independent of $\gamma$, $h$, and $f$.

To prove this estimate we invoke Lemmas 4.2 and 6.1:

$$
\begin{aligned}
\left\langle h, \frac{\partial^{|\beta|}}{\partial z^{\beta}} S_{\gamma}\left(P_{0} f\right)\right\rangle_{0} & =\left\langle\frac{\partial^{|\beta|}}{\partial z^{\beta}} M_{\beta} h, S_{\gamma}\left(P_{0} f\right)\right\rangle_{0} \\
& =\left\langle\frac{\partial^{|\beta|}}{\partial z^{\beta}} S_{\gamma+\beta}\left(M_{\beta} h\right), P_{0} f\right\rangle_{0} \\
& =\left\langle\frac{\partial^{|\beta|}}{\partial z^{\beta}} S_{\gamma+\beta}\left(M_{\beta} h\right), f\right\rangle_{0}=\left\langle S_{\gamma+\beta}\left(M_{\beta} h\right), L^{\beta} f\right\rangle_{0}
\end{aligned}
$$

where $L^{\beta}=L_{1}^{\beta_{1}} \cdots L_{n}^{\beta_{n}}$. In modulus this is at most the norm of $M_{\beta}$ : $H^{0} \rightarrow H^{0}$ times the norm of $L^{\beta}: W^{r} \rightarrow W^{0}$ times $\|h\|_{0}\|f\|_{r}$. This proves estimate (4.3).

We have shown that $P_{0}$ is bounded from $W^{r}$ to $H^{r}$ for integral $r \geq 0$. Just as in $\S 3$ it follows that

$$
\left[H^{s}, H^{r}\right]_{\theta}=H^{\theta r+(1-\theta) s}, \quad 0 \leq r \leq s, 0 \leq \theta \leq 1,
$$

and that $P_{0}$ is bounded from $W^{r}$ to $H^{r}$ for all real $r \geq 0$. Since the proof of Theorem 3.1(b) made no use of $\bar{\partial}$ methods beyond these facts, we may as well deduce Theorem 4.1(b) from the same proof. It is possible instead to prove Theorem 4.1(b) by the same method used to prove Theorem 4.1(a), introducing an operator $M_{\beta, s}$ analogous to $M_{\beta}$.

5. Moment inequalities in convex domains. This section is independent of the previous ones and involves only real analysis. It is devoted to the proof of the following theorem, central to the proof of Lemma 4.2. 
THEOREM 5.1. Let $G \subset \mathbf{R}_{+}^{n}$ be a bounded convex domain. For every quadruple of vectors $a, b, c, d$ in $\mathbf{R}_{+}^{n}$ such that $a+b=c+d$ we have the inequality

$$
\int_{G} x^{a} d m_{n} \int_{G} x^{b} d m_{n} \leq 4^{n|a-b|} \int_{G} x^{c} d m_{n} \int_{G} x^{d} d m_{n}
$$

where

$$
|a-b|=\sum_{j=1}^{n}\left|a_{j}-b_{j}\right|
$$

The constant on the right-hand side of (5.1) is not the best possible, but it is an easy one to write. In the application the precise value of the constant is irrelevant, but it is important that it depends only on the difference $|a-b|$.

Our approach to estimating the integrals involved in (5.1) is first to study integrals over $(n-1)$-dimensional slices. Fixing an arbitrary $(n-1)$-tuple $\rho=\left(\rho_{1}, \ldots, \rho_{n-1}\right)$ of nonnegative real numbers we define $I(Y)=\int_{Y} y^{\rho} d m_{n-1}$ for any measurable set $Y \subset \mathbf{R}_{+}^{n-1}$.

Lemma 5.2. Let measurable sets $Y_{0}$ and $Y_{1}$ in $\mathbf{R}_{+}^{n-1}$ be given. For each fixed $\rho$ we have

$$
I\left(Y_{0}\right)^{\lambda} I\left(Y_{1}\right)^{1-\lambda} \leq I\left(\lambda Y_{0}+(1-\lambda) Y_{1}\right)
$$

for all $\lambda \in(0,1)$.

Proof. We follow exactly the scheme of the proof of the BrünMinkowski theorem in [11, page 97]. By a standard approximation argument we can reduce to the case in which each of $Y_{0}$ and $Y_{1}$ admits a decomposition into a finite number of closed parallelepipeds with faces parallel to the coordinate hyperplanes, the interiors of the parallelepipeds being mutually disjoint.

Suppose to begin that each of $Y_{0}$ and $Y_{1}$ is a single parallelepiped. Denoting the coordinates of the vertices of $Y_{0}$ by $A_{J}$ and $B_{J}, 0 \leq A_{J}<B_{J}$, those of $Y_{1}$ by $C_{J}$ and $D_{J}, 0 \leq C_{J}<D_{J}, j=1, \ldots, n-1$, and setting $\rho_{j}+1=\sigma_{j}$, we compute

$$
\begin{aligned}
I\left(Y_{0}\right) & =\prod_{1}^{n-1} \sigma_{j}^{-1}\left(B_{j}^{\sigma_{J}}-A_{j}^{\sigma_{J}}\right), \quad I\left(Y_{1}\right)=\prod_{1}^{n-1} \sigma_{j}^{-1}\left(D_{j}^{\sigma_{J}}-C_{j}^{\sigma_{J}}\right), \\
I\left(\lambda Y_{0}+(1-\lambda) Y_{1}\right) & \\
& =\prod_{1}^{n-1} \sigma_{J}^{-1}\left[\left(\lambda B_{j}+(1-\lambda) D_{\jmath}\right)^{\sigma_{J}}-\left(\lambda A_{j}+(1-\lambda) C_{j}\right)^{\sigma_{\jmath}}\right] .
\end{aligned}
$$


Therefore to prove (5.2) it is enough to show

$$
\left(B^{\sigma}-A^{\sigma}\right)^{\lambda}\left(D^{\sigma}-C^{\sigma}\right)^{1-\lambda} \leq(\lambda B+(1-\lambda) D)^{\sigma}-(\lambda A+(1-\lambda) C)^{\sigma}
$$

for $0 \leq A<B, 0 \leq C<D, 0<\lambda<1, \sigma \geq 1$. It is equivalent to show that the function $\varphi(x, y)=(x+y)^{\sigma}-y^{\sigma}, \sigma \geq 1$, is logarithmically concave for $(x, y) \in \mathbf{R}_{+}^{2}$. (We recall that a continuous non-negative function $f$ defined in a convex region in Euclidean space is called logarithmically concave if $f(u)^{\lambda} f(v)^{1-\lambda} \leq f(\lambda u+(1-\lambda) v)$ for all $\lambda \in(0,1)$ and all $u, v$ in its domain.)

We show in fact something stronger: The function $\psi(x, y)=$ $\varphi(x, y)^{1 / \sigma}$ is concave. To show this we must check that the Hessian matrix of $\psi$ is negative (semi-)definite. A computation gives

$$
\begin{gathered}
\psi_{x x}=-y^{\sigma} \chi(x, y), \quad \psi_{y y}=-x^{2} y^{\sigma-2} \chi(x, y), \\
\psi_{x y}=\psi_{y x}=x y^{\sigma-1} \chi(x, y),
\end{gathered}
$$

where

$$
\chi(x, y)=(\sigma-1)(x+y)^{\sigma-2} \varphi(x, y)^{1 / \sigma-2} .
$$

Thus

$$
\psi_{x x} \leq 0, \quad \psi_{y y} \leq 0, \text { and } \psi_{x x} \psi_{y y}-\psi_{x y} \psi_{y x}=0
$$

which proves the result.

Next we assume inductively that the result holds when the total number of parallelepipeds in $Y_{0}$ and $Y_{1}$ is less than $k$, and suppose that $Y_{0}$ and $Y_{1}$ together are composed of $k$ parallelepipeds. We may assume that $Y_{0}$ contains at least two of them. There is some hyperplane, say $y_{j}=\mu$, such that some parallelepiped of $Y_{0}$ has no points in $y_{j}<\mu$ and some parallelepiped of $Y_{0}$ has no points in $y_{j}>\mu$. Let $\Gamma_{1}$ denote those parallelepipeds and parts of parallelepipeds in $y_{j}<\mu$ and $\Gamma_{2}$ those in $y_{j}>\mu$. Similarly split $Y_{1}$ into $\Delta_{1}$ and $\Delta_{2}$ by the hyperplane $y_{j}=\nu$, where $\nu$ is chosen to ensure that

$$
\frac{I\left(\Gamma_{1}\right)}{I\left(\Delta_{1}\right)}=\frac{I\left(\Gamma_{2}\right)}{I\left(\Delta_{2}\right)}=\frac{I\left(Y_{0}\right)}{I\left(Y_{1}\right)}
$$

The induction hypothesis applies to each of the pairs $\Gamma_{1}, \Delta_{1}$ and $\Gamma_{2}, \Delta_{2}$. Observing that $\lambda \Gamma_{1}+(1-\lambda) \Delta_{1}$ and $\lambda \Gamma_{2}+(1-\lambda) \Delta_{2}$ are disjoint, we find

$$
\begin{aligned}
I\left(\lambda Y_{0}+(1-\lambda) Y_{1}\right) & \geq I\left(\lambda \Gamma_{1}+(1-\lambda) \Delta_{1}\right)+I\left(\lambda \Gamma_{2}+(1-\lambda) \Delta_{2}\right) \\
& \geq I\left(\Gamma_{1}\right)^{\lambda} I\left(\Delta_{1}\right)^{1-\lambda}+I\left(\Gamma_{2}\right)^{\lambda} I\left(\Delta_{2}\right)^{1-\lambda} \\
& =I\left(Y_{0}\right)^{\lambda} I\left(Y_{1}\right)^{1-\lambda} .
\end{aligned}
$$

This completes the induction and proves the lemma. 
LEMMA 5.3. [13, 17, p. 77] If $f$ is a non-negative logarithmically concave function of $z \in[0, \infty)$ then

$$
\frac{1}{\Gamma(r+1)} \int_{0}^{\infty} z^{r} f(z) d z
$$

is a logarithmically concave function of $r \in[0, \infty)$.

LEMMA 5.4. (i) The function $\Gamma(z+1) / z^{z}$ is logarithmically concave in $z \in(0, \infty)$.

(ii) For $A \geq 0$ we have

$$
\Gamma(1+A) \leq 2^{A} \Gamma(1+A / 2)^{2} .
$$

Proof. We remind the reader how to prove these fairly standard facts. The formula

$$
\frac{d^{2}}{d z^{2}} \log \Gamma(1+z)=\sum_{k=1}^{\infty} \frac{1}{(z+k)^{2}}<\frac{1}{z}
$$

for $z>0$ yields statement (i).

Applying (i) on the interval $[0, A]$ (which requires invoking continuity of $\Gamma(z+1) / z^{z}$ at the left-hand endpoint) we get

$$
\frac{\Gamma(1)}{1} \frac{\Gamma(1+A)}{A^{A}} \leq \frac{\Gamma(1+A / 2)^{2}}{(A / 2)^{A}}
$$

which is statement (ii).

Proof of Theorem 5.1. We denote points $x \in G$ by $x=(y, z)$ where $y \in \mathbf{R}_{+}^{n-1}$ and $z \in \mathbf{R}_{+}$. Let $G(z)=\left\{y \in \mathbf{R}_{+}^{n-1}:(y, z) \in G\right\}$. Since by hypothesis $G$ is a convex domain,

$$
\lambda G(z)+(1-\lambda) G\left(z^{\prime}\right) \subset G\left(\lambda z+(1-\lambda) z^{\prime}\right) .
$$

It then follows by Lemma 5.2 that

$$
f(z)=\int_{G(z)} y^{\rho} d m_{n-1}
$$

is a logarithmically concave function of $z \in[0, \infty)$ for each fixed $\rho$. (Of course $f(z)=0$ for all large $z$.) 
Observe that if $f(z)$ is logarithmically concave on $[0, \infty)$ so is the function $z^{m} f(z)$ for every $m \geq 0$. Hence it follows by Lemmas 5.3 and 5.4 that for $0 \leq a_{n} \leq b_{n}$

$$
\begin{array}{rl}
\int_{G} y^{\rho^{a_{n}}} & d m_{n} \int_{G} y^{\rho} z^{b_{n}} d m_{n} \\
& =\int_{0}^{\infty}\left(z^{a_{n}} f(z)\right) d z \int_{0}^{\infty} z^{b_{n}-a_{n}}\left(z^{a_{n}} f(z)\right) d z \\
& \leq \frac{\Gamma(1) \Gamma\left(1+b_{n}-a_{n}\right)}{\Gamma\left(1+\left(b_{n}-a_{n}\right) / 2\right)^{2}}\left(\int_{0}^{\infty} z^{\left(b_{n}-a_{n}\right) / 2}\left(z^{a_{n}} f(z)\right) d z\right)^{2} \\
& \leq 2^{b_{n}-a_{n}}\left(\int_{G} y^{\rho} z^{\left(a_{n}+b_{n}\right) / 2} d m_{n}\right)^{2} .
\end{array}
$$

The analogous statement holds equally for each of the other variables.

Let us write $J(a)=\int_{G} x^{a} d m_{n}$. We have just shown that if the $n$-tuples $a$ and $b$ differ only in the $j$ th place then

$$
J(a) J(b) \leq 2^{\left|a_{\jmath}-b_{j}\right|} J((a+b) / 2)^{2} .
$$

Let us assume inductively that if $a$ and $b$ differ in at most $2^{k-1}$ places (where $k \geq 1$ is some integer such that $2^{k} \leq n$ ) then

$$
J(a) J(b) \leq J((a+b) / 2)^{2} 2^{2^{k-1}|a-b|} .
$$

Inequality (5.3) provides the basis step $k=1$. Suppose now that $a$ and $b$ differ in at most $2^{k}$ places, say the first $2^{k}$ places. Let $a^{\prime} \in \mathbf{R}_{+}^{n}$ agree with $a$ except in the first $2^{k-1}$ places, where $a^{\prime}$ agrees with $b$, and let $b^{\prime}$ agree with $b$ except in the first $2^{k-1}$ places, where $b^{\prime}$ agrees with $a$. Applying the induction hypothesis (5.4) three times we find

$$
\begin{aligned}
J(a) J(b) & \leq J\left(\left(a+a^{\prime}\right) / 2\right)^{2} J\left(\left(b+b^{\prime}\right) / 2\right)^{2} J\left(a^{\prime}\right)^{-1} J\left(b^{\prime}\right)^{-1} 2^{2^{k-1}\left(\left|a-a^{\prime}\right|+\left|b-b^{\prime}\right|\right)} \\
& \leq J((a+b) / 2)^{4} J\left(a^{\prime}\right)^{-1} J\left(b^{\prime}\right)^{-1} 2^{2^{k}|a-b|} \\
& \leq J((a+b) / 2)^{2} 2^{2^{k}|a-b|}
\end{aligned}
$$

where the last step follows by the Cauchy-Schwarz inequality. By induction we conclude that for arbitrary $a$ and $b$

$$
J(a) J(b) \leq J((a+b) / 2)^{2} 2^{n|a-b|}
$$

if $n$ is a power of 2 .

If $n$ is not a power of 2 we increase the dimension by taking the Cartesian product of the domain $G$ with a suitable number of copies of 
the unit interval. Since there is a power of 2 between $n$ and $2 n$, we conclude from (5.5) that

$$
J(a) J(b) \leq J((a+b) / 2)^{2} 2^{2 n|a-b|}
$$

for all $n$. Finally, observe that if $a+b=c+d$ then

$$
J((a+b) / 2)^{2}=J((c+d) / 2)^{2} \leq J(c) J(d)
$$

by the Cauchy-Schwarz inequality, so (5.1) follows. This completes the proof of Theorem 5.1.

Proof of Theorem 4.3. Let

$$
G=\left\{\left(x_{1}, \ldots, x_{n}\right):\left(x_{1}, \ldots, x_{n}\right)=\left(\left|z_{1}\right|, \ldots,\left|z_{n}\right|\right), z \in \Omega\right\} .
$$

Then

$$
\left\|z^{\gamma}\right\|_{0}^{2}=\int_{\Omega}\left|z^{\gamma}\right|^{2} d m_{2 n}=(2 \pi)^{n} \int_{G} x^{2 \gamma+1} d m_{n}
$$

where $2 \gamma+1$ denotes the multi-index $\left(2 \gamma_{1}+1, \ldots, 2 \gamma_{n}+1\right)$. Since $\Omega$ is a convex Reinhardt domain, $G$ is convex. Hence Theorem 4.3 follows from Theorem 5.1.

6. Holomorphic integration by parts. The idea behind this lemma is that tangential derivatives contribute no boundary terms upon integration by parts, and by the Cauchy-Riemann equations normal derivatives of holomorphic functions can be rewritten as tangential derivatives.

LEMMA 6.1. Let $\Omega \subset \mathbf{C}^{n}$ be any smooth bounded domain. There are first-order linear differential operators $L_{j}, j=1, \ldots, n$, with $C^{\infty}(\bar{\Omega})$ coefficients, such that

$$
\left\langle f, \partial h / \partial z_{j}\right\rangle_{0}=\left\langle L_{j} f, h\right\rangle_{0}
$$

for all $f \in W^{1}(\Omega)$ and all holomorphic $h \in H^{1}(\Omega)$.

Proof. We may assume $f \in C^{\infty}(\bar{\Omega})$, since this space is dense in $W^{1}(\Omega)$. Via a partition of unity we reduce to the case of $f$ with small support. If $f$ has compact support there is no difficulty, so we may assume supp $f \subset U$ where $U$ is a small neighborhood (in $\mathbf{C}^{n}$ ) of a boundary point $p \in b \Omega$.

If $\mathcal{E}$ is any tangential derivative in $U$ (that is, $\varrho \rho=0$ where $\rho$ is a defining function for $\Omega$ ) then $\langle f, \varrho h\rangle_{0}=\left\langle\varrho^{\prime} f, h\right\rangle_{0}$ where $\varrho^{\prime}$ is a differential operator of the same order as $\varrho$ containing lower order terms involving derivatives of the coefficients of $\varrho$ and derivatives of the smooth 
mapping which flattens the boundary near $p$. If $U$ is so small that for some $k$ we have $\partial \rho / \partial z_{k} \neq 0$ on $U$, then we can construct in $U$ smoothly varying orthonormal holomorphic vector fields $\mathfrak{L}_{1}, \ldots, \mathfrak{L}_{n}$ such that $e_{1}, \ldots, \mathscr{L}_{n-1}$, and $e_{n}+\overline{\mathscr{L}}_{n}$ are tangential to the boundary. Say $\partial / \partial z_{j}=$ $\sum_{i=1}^{n} c_{i j} \mathcal{L}_{i}$ in $U$ with $c_{i j} \in C^{\infty}(\bar{\Omega})$. Then since $h$ is holomorphic

$$
\frac{\partial h}{\partial z_{j}}=\left(c_{n j}\left(\varrho_{n}+\bar{\varrho}_{n}\right)+\sum_{i=1}^{n-1} c_{i j} \varrho_{l}\right) h=\varrho h
$$

where $e$ is a tangential derivative. Hence the lemma follows by the preceding remark.

\section{REFERENCES}

[1] N. Aronszajn, Theory of reproducing kernels, Trans. Amer. Math. Soc., 68 (1950), 337-404.

[2] D. Barrett, Regularity of the Bergman projection on domains with transverse symmetries, Math. Ann., 258 (1982), 441-446.

[3] S. R. Bell, Biholomorphic mappings and the $\bar{\partial}$-problem, Annals of Math., 114 (1981), 103-113.

[4] A representation theorem in strictly pseudoconvex domains, Illinois J. Math., 26 (1982), 19-26.

[5] S. R. Bell and H. P. Boas, Regularity of the Bergman projection in weakly pseudoconvex domains, Math. Ann., 257 (1981), 23-30.

[6] S. R. Bell and D. Catlin, Boundary regularity of proper holomorphic mappings, Duke Math. J., 49 (1982), 385-396.

[7] J. Bergh and J. Löfström, Interpolation Spaces, Springer-Verlag, 1976.

[8] S. Bergman, The Kernel Function and Conformal Mapping, 2nd ed., Providence: Amer. Math. Soc., 1970.

[9] D. Catlin, Boundary behavior of holomorphic functions on pseudoconvex domains, J. Differential Geometry, 15 (1980), 605-625.

[10] , personal communication.

[11] H. G. Eggleston, Convexity, Cambridge University Press, 1958.

[12] C. Fefferman, The Bergman kernel and biholomorphic mappings of pseudoconvex domains, Invent. Math., 26 (1974), 1-65.

[13] S. Karlin, Total Positivity, Stanford: Stanford University Press, 1968.

[14] J. J. Kohn, Global regularity of $\bar{\partial}$ on weakly pseudoconvex manifolds, Trans. Amer. Math. Soc., 181 (1973), 273-292.

[15] S. Krantz, Function Theory of Several Complex Variables, New York: Wiley, 1982.

[16] J. L. Lions and E. Magenes, Non-Homogeneous Boundary Value Problems and Applications, Vol. I., Springer-Verlag, 1972.

[17] A. W. Marshall and I. Olkin, Inequalities: Theory of Majorization and its Applications, New York: Academic Press, 1979.

Received March 9, 1982 and in revised form December 20, 1982. This material partially based on work supported by the National Science Foundation under Grant No. MCS8201063. 


\title{
PACIFIC JOURNAL OF MATHEMATICS EDITORS
}

\author{
Donald BabBITT (Managing Editor) \\ University of California \\ Los Angeles, CA 90024 \\ Hugo Rossi \\ University of Utah \\ Salt Lake City, UT 84112 \\ C. C. Moore and Arthur Ogus \\ University of California \\ Berkeley, CA 94720
}

\author{
J. Dugundu \\ Department of Mathematics \\ University of Southern California \\ Los Angeles, CA 90089-1113
}

R. FINN and H. SAMELSON

Stanford University

Stanford, CA 94305

\section{ASSOCIATE EDITORS}
R. ARENS
E. F. BECKENBACH
B. H. NeumanN
F. WolF
K. YosHIDA (1906-1982)

\section{SUPPORTING INSTITUTIONS}

\author{
UNIVERSITY OF ARIZONA \\ UNIVERSITY OF BRITISH COLUMBIA \\ CALIFORNIA INSTITUTE OF TECHNOLOGY \\ UNIVERSITY OF CALIFORNIA \\ MONTANA STATE UNIVERSITY \\ UNIVERSITY OF NEVADA, RENO \\ NEW MEXICO STATE UNIVERSITY \\ OREGON STATE UNIVERSITY
}

\author{
UNIVERSITY OF OREGON \\ UNIVERSITY OF SOUTHERN CALIFORNIA \\ STANFORD UNIVERSITY \\ UNIVERSITY OF HAWAII \\ UNIVERSITY OF TOKYO \\ UNIVERSITY OF UTAH \\ WASHINGTON STATE UNIVERSITY \\ UNIVERSITY OF WASHINGTON
}

The Supporting Institutions listed above contribute to the cost of publication of this Journal, but they are not owners or publishers and have no responsibility for its content or policies.

Mathematical papers intended for publication in the Pacific Journal of Mathematics should be in typed form or offset-reproduced (not dittoed), double spaced with large margins. Please do not use built up fractions in the text of the manuscript. However, you may use them in the displayed equations. Underline Greek letters in red, German in green, and script in blue. The first paragraph must be capable of being used separately as a synopsis of the entire paper. In particular it should contain no bibliographic references. Please propose a heading for the odd numbered pages of less than 35 characters. Manuscripts, in triplicate, may be sent to any one of the editors. Please classify according to the scheme of Math. Reviews, Index to Vol. 39. Supply name and address of author to whom proofs should be sent. All other communications should be addressed to the managing editor, or Elaine Barth, University of California, Los Angeles, California 90024.

There are page-charges associated with articles appearing in the Pacific Journal of Mathematics. These charges are expected to be paid by the author's University, Government Agency or Company. If the author or authors do not have access to such Institutional support these charges are waived. Single authors will receive 50 free reprints; joint authors will receive a total of 100 free reprints. Additional copies may be obtained at cost in multiples of 50 .

The Pacific Journal of Mathematics is issued monthly as of January 1966. Regular subscription rate: $\$ 132.00$ a year (6 Vol., 12 issues). Special rate: $\$ 66.00$ a year to individual members of supporting institutions.

Subscriptions, orders for numbers issued in the last three calendar years, and changes of address should be sent to Pacific Journal of Mathematics, P.O. Box 969, Carmel Valley, CA 93924, U.S.A. Old back numbers obtainable from Kraus Periodicals Co., Route 100, Millwood, NY 10546.

The Pacific Journal of Mathematics ISSN 0030-8730 is published monthly by the Pacific Journal of Mathematics at P.O. Box 969, Carmel Valley, CA 93924. Application to mail at Second-class postage rates is pending at Carmel Valley, California, and additional mailing offices. Postmaster: Send address changes to Pacific Journal of Mathematics, P. O. Box 969, Carmel Valley, CA 93924.

PUBLISHED BY PACIFIC JOURNAL OF MATHEMATICS. A NON-PROFIT CORPORATION

Copyright $\odot 1984$ by Pacific Journal of Mathematics 


\section{Pacific Journal of Mathematics}

\section{Vol. 112, No. $2 \quad$ February, 1984}

Kenneth F. Andersen and Wo-Sang Young, On the reverse weak type inequality for the Hardy maximal function and the weighted classes

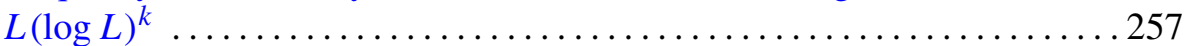

Richard Eugene Bedient, Double branched covers and pretzel knots ..... 265

Harold Philip Boas, Holomorphic reproducing kernels in Reinhardt

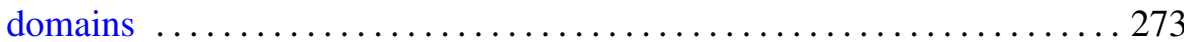

Janey Antonio Daccach and Arthur Gabriel Wasserman, Stiefel's theorem and toral actions ................................. 293

Michael Fried, The nonregular analogue of Tchebotarev's theorem ....... 303

Stanley Joseph Gurak, Minimal polynomials for circular numbers . . . . . . 313

Norimichi Hirano and Wataru Takahashi, Nonlinear ergodic theorems for an amenable semigroup of nonexpansive mappings in a Banach space . . 333

Jim Hoste, Sewn-up $r$-link exteriors . . . . . . . . . . . . . . . . . . . 347

Mohammad Ahmad Khan, The existence of totally dense subgroups in

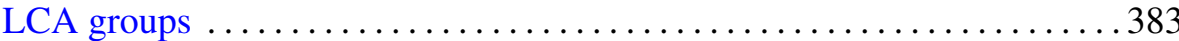

Mieczysław Kula, Murray Angus Marshall and Andrzej Sładek, Direct

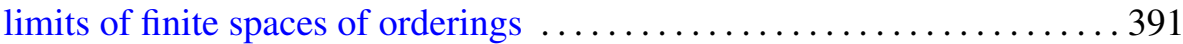

Luis Montejano Peimbert, Flat Hilbert cube manifold pairs ........... 407

Steven C. Pinault, An a priori estimate in the calculus of variations . . . . . 427

McKenzie Y. K. Wang, Some remarks on the calculation of Stiefel-Whitney classes and a paper of Wu-Yi Hsiang's

Brian Donald Wick, The calculation of an invariant for Tor . . 445

Wolfgang Wollny, Contributions to Hilbert's eighteenth problem 